\title{
P069: Drug-resistant acinetobacter ventilator-associated pneumonia: a time for desperate measures!
}

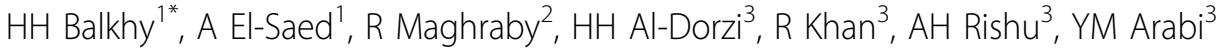 \\ From 2nd International Conference on Prevention and Infection Control (ICPIC 2013) \\ Geneva, Switzerland. 25-28 June 2013
}

\section{Introduction}

There is a wide geographic and temporal variability of bacterial resistance among microbial causes of ventilatorassociated pneumonia (VAP). The contribution of multidrug resistant (MDR) pathogens to the VAP etiology in Saudi Arabia was never studied.

\section{Objectives}

We sought to examine the extent of multiple-drug resistance among common microbial causes of VAP.

\section{Methods}

We conducted a retrospective susceptibility study in the adult ICU of King Abdulaziz Medical City, Riyadh, Saudi Arabia. Susceptibility results of isolates from patients diagnosed with VAP between October 2004 and June 2009 were examined. The US National Healthcare Safety Network (NHSN) definition of MDR was adopted.

\section{Results}

A total of 248 isolates including 9 different pathogens were included.Acinetobacter spp. was highly (70-90\%) resistant to all tested antimicrobials including carbapenems (three- and four-class MDR prevalence were 86\% and $78 \%$, respectively). Pseudomonas aeruginosa was moderately (20-40\%) resistant to all tested antimicrobials including antipseudomonal penicillins(three- and fourclass MDR prevalence were $18 \%$ and $10 \%$, respectively). With exception of ampicillin (fully resistant), Klebsiella spp. had low (0-14\%) resistance to other tested antimicrobials with no detected MDR. Staphylococcus aureuswas fully susceptible to vancomycin with $42 \%$ resistance to oxacillin. There were significant increasing trends of MDR Acinetobacterspp. but not Pseudomonas aeruginosa during the study.

\section{Conclusion}

Acinetobacterin the current study was an increasingly resistant VAP-associated pathogen more than seen in many parts of the world. The current finding may impact local choice of initial empiric antibiotic and emphasize the need to improve currently implemented antimicrobial stewardship and environmental cleaning. Measures to reduce the burden of this organism from such sites may assist in reducing the burden of Acinetobacter as a human pathogen in healthcare settings.

\section{Disclosure of interest}

None declared.

\section{Author details}

'Department of Infection Prevention and Control, King Abdulaziz Medical City, Riyadh, Saudi Arabia. ${ }^{2}$ Department of Pediatrics, King Abdulaziz Medical City, Riyadh, Saudi Arabia. ${ }^{3}$ Intensive Care Department, King Abdulaziz Medical City, Riyadh, Saudi Arabia.

Published: 20 June 2013

doi:10.1186/2047-2994-2-S1-P69

Cite this article as: Balkhy et al: P069: Drug-resistant acinetobacter ventilator-associated pneumonia: a time for desperate measures!. Antimicrobial Resistance and Infection Control 2013 2(Suppl 1):P69. 